\title{
English Teaching Contests and Professional Development of English Teachers in Independent Colleges ---- An Empirical Study Based on C College
}

\author{
Feng Xinyan
}

City College of Wuhan University of Science and Technology, Wuhan, Hubei Province, China 529336509@qq.com

Keywords: Teaching contest, Independent college, Teacher professional development.

\begin{abstract}
Teacher professional development is an important aspect of teachers' team construction. With arise and development of national college English teaching contests, their great potential to promote the professional development of college English teachers gradually becomes a consensus. With C College as an example, this paper introduces a new approach, which accords with the characteristics of independent colleges and takes English teaching contests as the carrier, to promote teachers' professional development and explores the feasibility and effectiveness of this approach through analyzing investigation data obtained from C College.
\end{abstract}

\section{英语教学大赛促进独立学院英语教师专业发展 \\ ---以C学院为例的实证研究 \\ 冯新艳 \\ 武汉科技大学城市学院, 武汉, 湖北, 中国 \\ 529336509@qq.com}

关键词：教学大赛；独立学院；教师专业发展

中文摘要. 教师的专业发展是教师队伍建设的重要方面。随着全国性大学英语教学赛事的兴起, 英语 教学大赛可以促进教师的专业发展逐渐成为共识。本文以C学院为例, 介绍一条符合独立学院特点的, 以英语教学大赛为载体的促进教师专业发展的新途径, 并结合调查研究的数据探究此途径的可行性和 有效性。

\section{1. 引言}

2010年，《国家中长期教育改革和发展规划纲要（2010-2020）》颁布，强调教师素质是 提高教学质量的关键, 应注重提高教师专业技能, 提高教师素质, 发展教师潜力。在此背景 下, 我国诞生了第一个全国性大规模的大学英语教学大赛-- “外教社杯” 大学英语教学大赛, 第一届大赛便覆盖了全国28个省市自治区约一千多所高校近一万名教师（束定芳，2010）。 其它全国性赛事，如 “教学之星大赛” 和 “中国外语微课大赛” 于2013年和2014年先后问世, 大赛对英语教师专业发展的意义与作用开始受到越来越多的关注和肯定。戚亚军（2014）从 对话哲学视角分析和阐释了大赛教学的功能, 肯定了大赛教学对常态教学的辐射力度。

然而, 英语教学大赛在促进教师专业发展过程中究竟应该扮演怎样的角色? 如何科学发 挥其在教师发展过程中的作用? 其对教师专业发展的有效性如何验证? 国内相关的研究尚为 
空白。本文以C学院为例, 介绍一条符合独立学院特点的, 以英语教学大赛为载体的促进教师 专业发展的新途径, 并结合调查研究的数据探究此途径的可行性和有效性。

\section{2. 独立学院英语教师的专业发展}

目前, 国内关于英语教师专业发展的研究甚少, 且多泛泛提出 “加强在职教育; 积极开 展教学科研活动; 开展教师的自我进修” 等原则性建议（文秋芳等，2010；董金伟，2012）。 已经提出的大学英语教师专业发展途径主要包括自我反思型, 协同发展或同伴互助型以及两 者结合型等（秋杰，2013; 郭燕等，2015），对其他院校具有一定的借鉴意义。

由于独立学院历史不长, 师资力量相对薄弱, 教师的教科研能力有限等因素的影响, 有 关其教师专业发展的研究胗胗可数, 且均为单一的描述性研究（史云林等, 2015）。目前暂 未发现关于独立学院英语教师专业发展途径的研究。

现有相关独立学院英语教师专业发展的研究主要探讨独立学院英语教师专业发展方面存 在的问题和解决的对策。研究者们提出的问题归类如下（见表1）:

表1＼cjkstart独立学院英语教师专业发展的主要问题归类

\begin{tabular}{|c|c|c|c|c|}
\hline & 研究者发现的问题 & \multicolumn{3}{|c|}{ 问题归类 } \\
\hline 1 & “知识结构单一” , “知识结构不合理” & 知识 & \multirow{3}{*}{ 客观问题 } & \multirow{5}{*}{$\begin{array}{l}\text { 个 } \\
\text { 人 } \\
\text { 因 } \\
\text { 素 }\end{array}$} \\
\hline 2 & “职称水平偏低, 教学经验缺乏”, “教学能力与经验不足” & 教学能力 & & \\
\hline 3 & “科研水平较低”，“科研能力不够” & 科研能力 & & \\
\hline 4 & “教学理念落后”, “科研意识淡薄” & 教科研意识 & \multirow{2}{*}{ 主观问题 } & \\
\hline 5 & “专业化发展意识淡薄” & 自我发展意识 & & \\
\hline 6 & ““队伍结构不合理”，“队伍流动性大” & \multirow{2}{*}{\multicolumn{3}{|c|}{ 学校、社会因素 }} \\
\hline 7 & “缺少专业培训”，“教师培养和培训机制不完善” & & & \\
\hline
\end{tabular}

总体而言, 独立学院英语教师专业发展的问题主要涉及教师的知识、能力和意识三个方 面, 反映了教师专业发展的主要方面, 正如郄玉婷（2012）分析提出：教师专业发展的内涵 在国内外的 “具体提法虽各有不同，但基本都认为教师应具有的专业素质应包括专业知识、 专业能力、专业精神等”。

针对以上问题提出的对策主要包括：从教师自主性出发解决问题，激发自我发展意识， 优化知识结构; 努力提高自身学历和科研水平; 进行教学反思, 积累教学经验; 注重教师合 作, 发展互助教学 (孙爱娜等, 2014) 。此外, 沈琳 (2014) 强调英语教师们应主动学习并 掌握现代化教学技术和手段, 王连芬 (2015) 建议英语教师积极参加培训、比赛, 主动争取 进修机会。同时, 所有研究者均呼吁学校和社会完善专业化支持和保障体系, 建立有效的激 励机制, 帮助和引导教师实现自我发展, 并给出一些具体的措施建议, 诸如构建校本教育模 式、拓展教师培训渠道、加大校本培训力度和促进教师间的相互协作等。

综上所述，专业素质问题应该引起独立学院的英语教师和教学管理者注意，教学管理者 有责任采取切实可行的措施促进教师的专业发展。然而, 研究者们提出的具体措施如何落实, 举措是否有效, 尚待进一步探索和研究。

\section{C学院英语教师专业发展的新途径}

C学院是成立于 2004 年的独立学院，集体备课、听课评课等校本培训活动最初几年受到 老师们欢迎, 但之后老师们参与的积极性不断降低, 培训效果不断减弱, C学院亟需新的途 径促进教师的专业发展。借鉴分析其他院校采用的途径后, C学院结合学院实际和特点, 尝 试开拓一条以英语教学大赛为载体的促进教师专业发展的新途径: 针对教师实际水平和发展 需求, 将各级各类培训、讲座等活动有机融入到各类英语教学大赛的赛前、赛中和赛后三个 不同阶段, 以达到充分利用各方资源促进英语教师专业发展的目的。具体而言, 主要包括各 项大赛赛前发布赛事信息, 根据赛事特点确定合适的主题与形式开展培训, 鼓励动员教师参 
赛; 随后, 组织对参赛人员参赛作品2-3次的观摩和点评, 并根据教师表现或疑问穿插组织交 流、培训或讲座等; 鼓励其他青年教师前往比赛现场观摩学习; 赛后总结、分享、奖励, 学 习和讨论外语界专家的点评，开展更多有利于教师专业发展的活动等。

此途径具有以下六个特点:

(1) 短期目标与长远目标相结合。在此途径中, 组织活动的短期目标均是帮助英语教师 在大赛中获奖, 目标明晰, 对青年老师们具有较强的吸引力; 在实现每一个短期目标的过程 中, 各种精心设计的旨在提升教师专业素质的活动促使让学生受益的长远目标逐步得以实现。

(2) 开放性与针对性相结合。此途径并不强求老师参与每次的活动, 允许根据个人需求 选择性参与, 具有一定的开放性, 利于营造和谐自主的氛围; 活动同时兼具较强的针对性, 因为参赛者必然会全程参与, 与参赛者关系亲密的同事会经常参与, 活动的有效性得到保障。

(3) 校本培训与校外培训相结合。以全国性赛事为载体而开展的活动, 不仅本校直接培 训青年教师, 还可利用大赛主办方提供的各类讲座、研讨、音像或文字材料等, 让全国的其 他优秀教师、赛场评委、学者和专家们间接参与到培训本校青年教师的过程中, 有助于解决 独立学院高级职称教师少、专业指导老师人员不足水平有限等问题。校本培训利于增进老师 之间的感情, 校外培训利于扩展教师视野。

(4) 个人利益与集体利益相结合。教学大赛可以促使优秀老师们的能力得到认可和关注, 获得荣誉感和归宿感, 有助于自身专业发展。教师的荣誉也是集体的荣誉, 教师的成长便是 集体的成长, 集体的强大能增强其他老师的集体荣誉感, 有助于形成积极向上的良好氛围, 实现集体利益的最大化。

（5）静态与动态相结合。在部分校本培训活动中，被培训者可以分为参赛者、指导者、 参赛者的朋友同事和观摩教师, 但各种身份并非静止不变的, 例如本次的参赛者下次可能担 任指导者的角色。这种动态变化利于调动各方参与者的积极主动性。

（6）方向性与指导性相结合。英语教学大赛具有周期性和延续性, 是全国性高层次的教 学改革和交流的平台, 利于参与者了解新形势下的改革动态, 掌握相应的教学理念、方法和 手段（戴玮栋，2010），对教学管理者结合其它校内、校际教学竞赛等建立科学且适合本校 教师需求的培训体系具有指导作用。

\section{C学院英语教师专业发展的新途径的实施效果}

\section{1 显性效果}

C学院自2012年实施新途径以来, 教学竞赛获奖的老师人数逐年增加, 荣获的校级以上 荣誉达到24项, 参加的各类校外专业竞赛涵盖了教学竞赛、微课大赛和多媒体课件大赛, 其 中, 在规模最大、影响最广、级别最高的全国外语教学大赛---- “外教社杯” 全国高校外语大 赛 --- 中, 本校老师曾获省级特等奖，代表省参加全国总决赛，并最终荣获全国二等奖。同 时, 英语老师们在课堂教学方面取得的成绩和进步也令人欣喜, 学生对英语老师的教学评价 优秀率近三年达到 $100 \%$, 学生研究生考试英语及格率（60分以上）人数年年上升, 从之前的 约 $20 \%$ 到达 2016 年的 $42 \%$ 。近两年, 英语教师们成功申报了 5 项省厅级教科研课题, 并完成了 一项省级精品课程。

以上这些成果展现了笔者所在院校英语教师的整体素质和水平, 也证明了英语教学大赛 服务独立学院英语教师的专业发展的可行性和效果。

\section{2 调查研究发现与讨论}

2016年底, 笔者利用问卷调查 $C$ 学院英语教师对英语教学大赛的认识等, 以期从教学管 理者的视角检视英语教学大赛对一线教师专业发展的有效性和应用价值, 为探索独立学院英 语教师专业发展的有效途径提供参考。

笔者发出40份问卷，回收36份有效问卷，问卷主要涉及以下问题:（1）你认为英语教师 是否有必要参加或者观摩各类专业竞赛?（2）你参加比赛的原因或者您认为其他英语教师参 
加比赛最主要的原因是什么?（3）你认为老师报名参赛后, 系（部）是否有责任对老师进行 指导?（4）在准备参赛的过程中，你（将）主要通过什么途径提高自己的作品质量?

你认为英语类教学竞赛（将）给老师带去的影响有哪些? 有参赛经历的老师基于个人经历回 答问卷，其他老师则基于个人观点回答。

\subsection{1 参与调查的教师的基本状况}

从年龄和参与 $\mathrm{C}$ 学院教师专业发展项目时间方面看, 参与调查的 36 名英语教师中, 50 岁 以上的 1 名，30-40岁之间的 24 名，占全部人数的 $66.7 \%$, 参加项目的时间达到 4 年; 30 岁以下 的有 11 名, 占全部人数的 $30.6 \%$, 参与教师专业发展项目不足 2 年。从参赛经历分析, 参与调 查的 36 名教师中有 13 人曾参加过校内或校外教学比赛, 6 人计划未来参加比赛, 达到全部参与 调查人员的 $52.8 \%$ 。从担任的角色分析, 有 8 名英语教师承担了系主任或教研室主任等教学管 理工作，具有双重身份。

\subsection{2 参加或观摩各类专业竞赛的必要性}

总体上，有 27 人 (占75\%) 认为英语教师非常有必要参加或观摩各类专业竞赛，9人（占 $25 \%$ ）认为应该由老师自己决定是否要参赛或观摩。其中，承担教学管理工作的 8 名教师全部 认为英语教师非常有必要参赛或观摩比赛, 而部分曾参赛的老师 (3人，23\%) 和计划参赛的 老师 (2人， $33 \%$ ) 则认为应该由老师自己决定是否参加。这提醒教学管理者, 尽管参加或观 摩专业竞赛有利于教师的发展，但是在组织相关活动时应注意避免出现行政干预或强迫的情 况，给予教师一定的自主选择权，以保证活动的最佳效果。

此外，30岁以上的老师对于必要性的认可程度（19人， 79.17\%) 高于30岁以下教师（7 人, $63.64 \%$ ）。这或许说明, 参加C学院英语教师专业发展项目时间越长的老师, 对竞赛的 认可程度越高。

\subsection{3 参加教学比赛的动机}

数据显示，老师们认为英语教师参加比赛主要是为了 “提升个人能力的意愿程度”（30 人, $80.33 \%)$ ， “职称评审” (28人, $77.78 \%$ ) 和 “对个人能力的自信度” (20人, $55.56 \%)$ 。 从表2可见，30-40岁的教师中选择 “提升个人能力的意愿程度” 的人 (23人，95.8\%）多于选 择 “职称评审” 的人 (18人， $75 \%)$ ，而30岁以下教师中选择 “职称评审” 的人 (10人，91\%) 明显多于选择 “提升个人能力的意愿程度” 的人（6人， $54.6 \%$ ）。

表2 不同年龄教师参加教学比赛的动机

\begin{tabular}{|l|l|l|l|l|l|l|l|l|}
\hline $\mathrm{X} \backslash \mathrm{Y}$ & $\begin{array}{l}\text { 对个人能 } \\
\text { 力的自信 } \\
\text { 度 }\end{array}$ & $\begin{array}{l}\text { 个人的兴 } \\
\text { 趣 }\end{array}$ & $\begin{array}{l}\text { 提升个人能 } \\
\text { 力的意愿程 } \\
\text { 度 }\end{array}$ & 职称评审 & $\begin{array}{l}\text { 领导的期 } \\
\text { 望或要求 }\end{array}$ & 年度考核 & 其它 & $\begin{array}{l}\text { 小 } \\
\text { 计 }\end{array}$ \\
\hline 30 岁以下 & $5(45.45 \%)$ & $5(45.45 \%)$ & $6(54.55 \%)$ & $10(90.91 \%)$ & $5(45.45 \%)$ & $2(18.18 \%)$ & $0(0.00 \%)$ & 11 \\
\hline $30--40$ 岁 & $14(58.33 \%)$ & $12(50.00 \%)$ & $23(95.83 \%)$ & $18(75.00 \%)$ & $2(8.33 \%)$ & $3(12.50 \%)$ & $0(0.00 \%)$ & 24 \\
\hline $40--50$ 岁 & $0(0.00 \%)$ & $0(0.00 \%)$ & $0(0.00 \%)$ & $0(0.00 \%)$ & $0(0.00 \%)$ & $0(0.00 \%)$ & $0(0.00 \%)$ & 0 \\
\hline 50 岁以上 & $1(100.00 \%)$ & $1(100.00 \%)$ & $1(100.00 \%)$ & $0(0.00 \%)$ & $0(0.00 \%)$ & $0(0.00 \%)$ & $0(0.00 \%)$ & 1 \\
\hline
\end{tabular}

笔者分析，这并不意味着30-40岁的老师不关注职称评审，实际上“职称评审”是他们参赛 的第二大诱因; 他们的选择与 30 岁以下教师的选择的差异很可能源于参与 $\mathrm{C}$ 学院教师专业发 展项目时间的长短。换言之, $\mathrm{C}$ 学院以英语教学大赛为依托的教师专业发展项目对于增强教 师的自我发展意识有很好效果和作用, 得到了教师们的认可。正如某位老师针对此问题所补 充的, “参赛者能发现自己在教学理论学习中的不足, 去思考并学习更多的教学方法, 并能 看到自己和其他老师之间的差距，加强自我提升的动力”。

4.2 .4 系（部）对报名参赛老师的指导责任

多数老师 (约 $80 \%$ ) 认为系 (部) 有责任和义务指导老师参赛, 承担教学管理任务的 8 名 老师则一致认为系 (部) 有此责任和义务。可见, C学院以赛为依托的专业发展项目已经深 入人心, 参与教学管理的老师们已经将赛事指导视为自己不容推辞的责任, 而老师们也期待 教学管理者们的指导和帮助。 


\subsection{5 备赛过程中的学习途径}

30 岁以下的教师最期待（10人，90.91\%）从系（部）获得有价值的建议，30-40岁之间的 教师则更多的选择（21人， $80.57 \%$ ）自行观摩学习相关课程。这一方面表明了 30 岁以下老师 对系 (部) 的信任和依赖, 另一方面也说明参加 $\mathrm{C}$ 学院以赛为依托的专业发展项目时间较长 的教师（30岁以上）具有更强的自我发展意识。

表3＼cjkstart英语教师备赛过程中的学习途径

\begin{tabular}{|l|l|l|l|l|l|l|l|l|}
\hline $\mathrm{X} \backslash \mathrm{Y}$ & $\begin{array}{l}\text { 完全靠个 } \\
\text { 人准备 }\end{array}$ & $\begin{array}{l}\text { 读了一些关 } \\
\text { 于教学理论 } \\
\text { 和教学方法 } \\
\text { 的文章或书 } \\
\text { 籍 }\end{array}$ & $\begin{array}{l}\text { 关亲密的 } \\
\text { 朋友或同事 } \\
\text { 给我提了一 } \\
\begin{array}{l}\text { 些有价值的 } \\
\text { 建议 }\end{array}\end{array}$ & $\begin{array}{l}\text { 所在系 (部) } \\
\text { 主动给予了 } \\
\text { 些有价值 } \\
\text { 的建议 }\end{array}$ & $\begin{array}{l}\text { 学生给了 } \\
\text { 我一些有 } \\
\text { 价值的建 } \\
\text { 议 }\end{array}$ & $\begin{array}{l}\text { 其他途径观 } \\
\text { 摩了一些相 } \\
\text { 关的课程 }\end{array}$ & $\begin{array}{l}\text { 不用准 } \\
\text { 备, 赤膊 } \\
\text { 上阵即可 }\end{array}$ & $\begin{array}{l}\text { 小 } \\
\text { 计 }\end{array}$ \\
\hline $\begin{array}{l}30 \text { 岁以 } \\
\text { 下者 }\end{array}$ & $1(9.09 \%)$ & $6(54.55 \%)$ & $6(54.55 \%)$ & $10(90.91 \%)$ & $2(18.18 \%)$ & $8(72.73 \%)$ & $0(0.00 \%)$ & 11 \\
\hline $\begin{array}{l}30--40 \\
\text { 岁者 }\end{array}$ & $5(20.83 \%)$ & $15(62.50 \%)$ & $15(62.50 \%)$ & $17(70.83 \%)$ & $7(29.17 \%)$ & $21(87.50 \%)$ & $0(0.00 \%)$ & 24 \\
\hline $\begin{array}{l}\text { 教学管 } \\
\text { 理者 }\end{array}$ & $0(0.00 \%)$ & $7(87.50 \%)$ & $5(62.50 \%)$ & $8(100.00 \%)$ & $2(25.00 \%)$ & $7(87.50 \%)$ & $0(0.00 \%)$ & 8 \\
\hline $\begin{array}{l}\text { 曾经参 } \\
\text { 赛者 }\end{array}$ & $2(15.38 \%)$ & $9(69.23 \%)$ & $9(69.23 \%)$ & $9(69.23 \%)$ & $4(30.77 \%)$ & $11(84.62 \%)$ & $0(0.00 \%)$ & 13 \\
\hline $\begin{array}{l}\text { 将要参 } \\
\text { 赛者 }\end{array}$ & $2(33.33 \%)$ & $4(66.67 \%)$ & $5(83.33 \%)$ & $4(66.67 \%)$ & $2(33.33 \%)$ & $3(50.00 \%)$ & $0(0.00 \%)$ & 6 \\
\hline
\end{tabular}

从表3可见，曾经参赛教师的真实学习经历和教学管理者及计划将来参赛的教师对学习的 预期并不一致。系（部）的建议对老师的学习意义受到普遍的认可，但是教学管理者似乎对 其作用略有高估: 参赛过的老师们更多地选择自主观摩课程, 而计划参赛的老师则期待从关 系亲密的同事和朋友处获得更多的帮助。

4.2.6 英语教学大赛对教师的影响

数据表明, 绝大多数老师认为英语教学大赛为老师提供了相互交流学习的机会, 增进了 同事之间的感情, 可从中学到有用的教学技巧。但是当绝大多数的 $30-40$ 岁教师 (20人, 83.33\%) 认为相关活动 “帮助强化教学目标意识和教学效果意识” 时, 30岁以下教师中仅有少数（5 人， $45.45 \%$ ）持相同看法。可见， C学院以赛为依托的专业发展项目对于增强教师队伍的凝 聚力, 增加教师的教学技巧有立竿见影的效果, 而要普遍提高教师的教学意识和教学能力则 需要更长的时间。

此外, 各类人群中, 认为大赛提升了自己教研兴趣的, 比例最高的是曾参赛的老师 (8 人， $61.54 \%$ ），其次是 $30-40$ 岁之间老师（11人， $45.83 \%$ ）。30岁以下的教师中仅有 $27.27 \%$ 的人 (3人) 的人有此体会。可见, 英语教学大赛 (或者 $\mathrm{C}$ 学院以赛为依托的专业发展项目) 对于提高老师的教研兴趣产生了一定的作用, 但是可能需要设计更多的活动培养和提升教师 的教科研意识和能力。

\section{5. 结束语}

经过四年的探索, $\mathrm{C}$ 学院实施的以英语教学大赛为载体的专业发展项目使得系（部）得 到了老师们的一致而高度的认可, 协作、融洽、积极向上的工作氛围已经形成, 充分证明了 项目的可行性; 问卷调查结果表明, 在项目的影响下, 教师们自主专业发展的意识明显增强, 教师更多关注自我提高和个人反思，项目的有效性得到了实证。我们有理由呼吁其他的独立 学院借鉴C学院的 “以英语教学大赛为载体的促进教师专业发展的新途径” 促进青年英语教 师的专业发展。 


\section{致谢}

本文为湖北省教育科学规划研究重点项目《独立学院英语教师专业发展的实证研究 ---- 基于 “以赛促教” 的尝试》(2015ZA015)的阶段性成果之一。

\section{References}

[1] Su Dingfang, College English classroom teaching, what we teach, how to teach? - closed remarks in the first SFLEP Cup National College English Teaching Contest, Foreign Language World, No.6, pp.26-32, 2010.

[2] Qi Yajun, From rough jade to fine jade: inquiry on the value of teaching effectiveness in the light of dialogical philogical -- based on classroom observation of the SFLEP Cup teaching contests and teaching in the regular classroom, Foreign Language World, No.4, pp.62-70+95, 2014.

[3] Wen Qiufang, Ren Qingmei, On in-service tertiary EFL teacher professional development in China: trends, characteristics, problems and solutions - by reviewing the related literature from 1999-2009, Foreign Language of China, No.4, pp.77-83, 2010.

[4] Dong Jinwei, On the learning strategies for college English teachers' professional development: an empirical study based on G university, Foreign Language Teaching Theory and Practice, No.2, pp. 14-22, 2012.

[5] Qiu Jie, Peer coaching and professional development of College English teachers, Thesis of Xi'an International Studies University, 2013.

[6] Guo Yan, Xu Jinfen, On the construction of professional development community of college English teachers, Foreign Language World, No.5, pp.79-87, 2015.

[7] Shi Yunlin, Gu Wei, Study on foreign language teaching theory of independent colleges -- by reviewing the related literature from 2004-2014, Education Teaching Forum, No.16, pp.218-221, 2015.

[8] Yan Yuting, Research on the young teachers' present situation of professional development and influence fators in Guangdong province independent college, Thesis of Guangdong University, 2012.

[9] Sun Aina, Li Yue, On the professional development of young college English teachers in independent colleges, Journal of Changchun Normal University, No.3, pp.143-145, 2014.

[10] Shen Lin, Research on professional development of independent college English teachers, Journal of Hubei University of Education, No.12, pp.112-114, 2014.

[11]Wang Lianfen, Present problems and solutions on professional development of English teachers in independent colleges, Journal of Kaifeng Institute of Education, No.11, pp.76-77, 2015.

[12]Dai Weidong, Set up a high level exchange platform on teaching reform to promote education and development of foreign language teachers, Foreign Language World, No.5, pp.7-8, 2010. 